\title{
DERECHO Y POLÍTICAS AMBIENTALES EN LA REGIÓN DE MURCIA (PRIMER SEMESTRE 2017)
}

\author{
SANTIAGO M. ÁLVAREZ CARREÑO \\ Profesor titular de Derecho Administrativo \\ Universidad de Murcia
}

\author{
ELISA PÉREZ DE LOS COBOS HERNÁNDEZ \\ Profesora ayudante doctor de Derecho Administrativo
}

Universidad de Murcia

\begin{abstract}
Sumario: 1. Introducción: sombras y luces de la política y legislación ambiental de la administración regional en Murcia. 1.1. Nuevos avances en la planificación de la Red Natura 2000. 1.2. La insensata e imparable destrucción de la laguna litoral del Mar Menor: el tiempo se acaba...2. Otras manifestaciones de la política y legislación ambiental de la administración regional en Murcia. 2.1. Educación ambiental. 2.2. Especies exóticas invasoras: el proyecto LIFE "Ripisilvanatura". 2.3. Hábitats de aves acuáticas en islas de la Región de Murcia. 2.4. Cambio climático. 2.5. Patrimonio natural arbóreo. 2.6. Pesca en aguas interiores. 2.7. Residuos: la aprobación del nuevo Plan de Residuos de la Región de Murcia 2016-2020. 2.8. Directiva Hábitats: modelos normalizados de comunicación previa de usos y actividades. 2.9. Participación ambiental: de nuevo sobre la destrucción del Mar Menor. 2.10. Flora: Plan de Recuperación del Garbancillo de Tallante.
\end{abstract}

1. INTRODUCCIÓN: SOMBRAS Y LUCES DE LA POLÍTICA Y LEGISLACIÓN AMBIENTAL DE LA ADMINISTRACIÓN REGIONAL EN MURCIA

La presente crónica sobre la política y legislación ambiental en la CARM, referida básicamente a este primer semestre del 2017, aunque también da cuenta de algunas aportaciones correspondientes a los últimos meses del año anterior, refleja ciertos avances positivos que deben ser convenientemente saludados. En especial, la aprobación de decretos que declaran nuevas zonas especiales de conservación y aprueban sus planes de gestión representa un paso decidido en la tutela de espacios naturales 
dentro de la Red Natura 2000 y, de alguna manera, viene a compensar los excesivos retrasos que se venían acumulando en este sector esencial para la protección ambiental.

Por otro lado, los efectos perjudiciales para la laguna salada del Mar Menor que se han ido acumulando durante décadas, derivados de la actividad agrícola en el Campo de Cartagena, además de la excesiva presión urbanística y turística, cristalizaron el pasado verano: el agua verdosa y maloliente por efecto de la eutrofización expulsaba a los veraneantes y provocaba la verdadera alarma que genera no la destrucción de una maravilla natural, sino las pérdidas económicas por la disminución de la actividad turística y la depreciación del valor de los inmuebles. Por desgracia, solo cuando estamos en una situación de verdadera emergencia ambiental se toman las medidas que se venían reclamando desde hace, por lo menos, veinte o más años. Esta crónica de una casi muerte anunciada de un espacio natural tan señero dejará constancia de una serie de medidas que se están adoptando y cuya eficacia empezará a demostrarse, en su caso, en los años venideros. En todo caso, si no se piensan alternativas al modelo de desarrollo económico implantado en la zona, tan dependiente del recurso agua, no conseguiremos mejoras sustanciales. Como señalan varios expertos, se debe reflexionar sobre lo que supone tener una agricultura intensiva e internacionalizada en la zona de mayor estrés hídrico de la UE1.

Hemos querido en la presente entrega de nuestra crónica subrayar la importancia de estas cuestiones dedicándoles sendos apartados en esta introducción. A continuación, referiremos otras manifestaciones, algunas de ellas también importantes, de la política y legislación ambiental en la CARM. Así, si en la anterior crónica dábamos cuenta de la aprobación del Decreto-Ley $2 / 2016$, de 20 de abril, de medidas urgentes para la reactivación de la actividad empresarial y del empleo a través de la liberalización y de la supresión de cargas burocráticas, aquí consignamos simplemente su conversión en la Ley 2/2017, de 13 de febrero (BOE, núm. 57, de 8 de marzo de 2017). Además, dejaremos constancia de la

\footnotetext{
${ }^{1}$ Vid. El País Negocios, 2 de abril de 2017, "Una economía cada vez más sedienta”, pp. 2-5.
} 
aprobación del nuevo Plan de Residuos regional y de algunas otras medidas que reflejan una mayor intensidad de la actuación ambiental de la Administración regional, consciente quizás del excesivo tiempo perdido en un ámbito en el que nos jugamos ya la calidad de vida de la ciudadanía de esta región.

\subsection{Nuevos avances en la planificación de la Red Natura 2000}

El Decreto 11/2017, de 15 de febrero, de declaración de la Zona Especial de Conservación (ZEC) de los Ríos Mula y Pliego, y aprobación de su plan de gestión (BORM, núm. 46, de 25 de febrero de 2017), supone la aprobación por el Consejo de Gobierno de la CARM del segundo Plan de Gestión de la Red Natura 2000. Así, la Región de Murcia cuenta ya con un nuevo espacio natural planificado, después del Noroeste, que está en vigor desde mayo de 2015. Este nuevo avance en la conservación del territorio afecta a una superficie de 829,73 hectáreas en la zona centro de la Región.

La CARM avanza lentamente en la tramitación de la Red Natura 2000, aunque todavía acumula un retraso de seis años con respecto a los plazos exigidos por la normativa comunitaria. La prensa regional informaba recientemente que el próximo plan de gestión en ser aprobado será el de Minas de la Celia (Jumilla)-Cueva de las Yeseras (Santomera), según había anunciado la propia consejera del ramo². Y, en efecto, el Decreto 13/2017, de 1 de marzo, declara las zonas especiales de conservación (ZEC) de las Minas de la Celia y la Cueva de las Yeseras, y aprueba su plan de gestión (BORM, núm. 64, de 18 de marzo de 2017)

Por otro lado, el Boletín Oficial de la Región (BORM), núm. 73, de 29 de marzo de 2017, publica tres anuncios de la Oficina de Impulso Socioeconómico del Medio Ambiente de la Consejería de Agua, Agricultura y Medio Ambiente por los que se somete a consulta pública previa la elaboración de los proyectos de decretos de aprobación de los planes de gestión integral de los espacios protegidos de los relieves y cuencas centro-

\footnotetext{
${ }^{2}$ Información accesible en http://lospiesenlatierra.laverdad.es/blog/4083-aprobada-la-zona-especial-deconservacion-de-los-rios-mula-y-pliego. (Fecha de acceso: 11/04/2017).
} 
orientales de la Región de Murcia, Saladares del Guadalentín y Sierra Espuña y su entorno.

Estos anuncios sobre la consulta pública de tres nuevos enclaves representan el trámite previo a la elaboración de los proyectos de decreto para aprobar los planes de gestión de dichos espacios protegidos. Los proyectos de decreto se corresponden con tres áreas de planificación integrada (API) de las catorce definidas para el conjunto de la CARM para dar coherencia a la planificación de los espacios protegidos de la Red Natura 2000. Conviene recordar que hasta el momento solo se han aprobado tres planes de gestión: el del Noroeste en 2015, el de los Ríos Mula y Pliego y el de las Minas de la Celia y la Cueva de las Yeseras, como se ha mencionado supra.

Con estos avances la Región de Murcia recupera en parte el retraso que sufren dichos trámites, cuya finalización se prevé para finales del año 2018.

\subsection{La insensata e imparable destrucción de la laguna litoral del Mar Menor: el tiempo se acaba...}

La gran preocupación generada por las manifestaciones eutróficas de la laguna costera del Mar Menor ha provocado la aprobación del Decreto-Ley $1 / 2017$, de 4 de abril, de medidas urgentes para garantizar la sostenibilidad ambiental en el entorno del Mar Menor (BORM, núm. 80, de 6 de abril de 2017).

La exposición de motivos del Decreto-Ley recuerda que el Mar Menor es una de las mayores lagunas litorales de Europa y la más grande de la península ibérica, con singulares valores ambientales que han comportado su incorporación en los Humedales de Importancia Internacional (RAMSAR) y Zonas Especialmente Protegidas de Importancia para el Mediterráneo (ZEPIM), así como la declaración del Paisaje Protegido de los Espacios Abiertos e Islas del Mar Menor, del Parque Regional de Salinas y Arenales de San Pedro del Pinatar, del Lugar de Importancia Comunitaria (LIC) "Mar Menor" y de la Zona de Especial Protección para las Aves (ZEPA) "Mar Menor". Sobre este espacio convergen múltiples usos y aprovechamientos, 
principalmente turísticos, recreativos y pesqueros, con un importante aprovechamiento agrícola de su entorno.

El grave deterioro de la calidad de sus aguas por la progresiva eutrofización de la laguna representa un problema de gran complejidad técnica, ambiental y social que exige actuar de forma combinada sobre los diferentes sectores de actividad que afectan a su estado ecológico. Existe una coincidencia sustancial en la comunidad científica sobre la necesidad de adoptar con urgencia medidas para evitar las principales afectaciones al Mar Menor; así, el Comité de Asesoramiento Científico del Mar Menor, en su "Informe integral sobre el estado ecológico del Mar Menor", de 13 de febrero de 2017, considera la contaminación por nitratos, que afecta también gravemente al acuífero cuaternario, como uno de los factores que ha contribuido al desequilibrio ambiental del Mar Menor, sin minusvalorar la contaminación por metales pesados o la procedente de aguas de escorrentías.

En cumplimiento de la Directiva 91/676/CEE del Consejo, de 12 de diciembre, relativa a la protección de las aguas contra la contaminación producida por nitratos utilizados en la agricultura, incorporada al ordenamiento jurídico español por el Real Decreto 26/1996, de 16 de febrero, buena parte del Campo de Cartagena ha sido declarada como zona vulnerable a la contaminación por nitratos y le es de aplicación el programa de actuación aprobado por la CARM. Es evidente que resulta necesario y urgente intensificar las acciones de protección, procurando una mayor sostenibilidad ambiental de las actividades que se realizan en el entorno del Mar Menor.

El presente Decreto-Ley se divide en cinco capítulos, que se completan con cinco disposiciones adicionales, una disposición transitoria, una disposición derogatoria y dos disposiciones finales. El capítulo I establece el objeto del Decreto-Ley (art. 1) y su ámbito de aplicación (art. 2), que se corresponde con la cuenca hidrográfica vertiente e incluye los municipios que tienen territorio en esta. Dentro de la cuenca, se distinguen tres zonas para establecer en ellas condiciones que aseguren la sostenibilidad ambiental de las explotaciones agrícolas en el Campo de Cartagena, de lo que se ocupa 
el capítulo II (arts. 3-11). Estas condiciones tienen por finalidad última la de preservar los recursos naturales y los valores ambientales del Mar Menor, y en especial los hábitats que han dado lugar a la designación del LIC "Mar Menor" y de la ZEPA "Mar Menor". La zona 1 comprende las explotaciones agrícolas situadas en la franja más próxima al Mar Menor (con exclusión de las zonas urbanas), por lo que las condiciones impuestas resultan más estrictas, estableciéndose en ella requerimientos adicionales que exigen implantar estructuras vegetales de barrera destinadas a la retención y regulación de aguas, y orientar los cultivos de manera adecuada para minimizar las escorrentías al Mar Menor. La zona 2 abarca la zona vulnerable correspondiente a los acuíferos cuaternario y plioceno en el área definida por zona regable oriental del Trasvase Tajo-Segura y litoral del Mar Menor en el Campo de Cartagena. La zona 3 se extiende por el resto de la cuenca vertiente. Seguidamente, el capítulo III (arts. 12-13), relativo al control de los vertidos al Mar Menor, establece que estos quedan prohibidos con carácter general. Los vertidos de aguas pluviales solo serán posibles en aquellos casos en que no resulte viable su eliminación por otros medios, debiendo los ayuntamientos realizar las inversiones encaminadas al cumplimiento de este objetivo, con el apoyo financiero de la Comunidad Autónoma que contempla la disposición adicional tercera.

Para la tramitación preferente y declaración de urgencia de las actuaciones relacionadas con los fines de la norma, el capítulo IV (arts. 14 a 16) prevé diversas medidas, también orientadas a clarificar los supuestos en que es exigible el trámite de evaluación ambiental en la actividad agrícola y a facilitar la expropiación forzosa de los bienes y derechos que puedan quedar afectados por las inversiones necesarias. La efectividad de todas estas limitaciones se asegura mediante el régimen sancionador y de control que desarrolla el capítulo $\mathrm{V}$ (arts. 17-22).

Finalmente, esta norma contiene dos importantes medidas, de especial importancia en las prácticas agrarias del Campo de Cartagena y que constituyen un complemento necesario para el cumplimiento de sus objetivos, pero que, por ser de aplicación a todo el ámbito territorial regional, aparecen como disposiciones adicionales: la aprobación de un 
nuevo Código de Prácticas Agrícolas de la Región de Murcia, que se inserta en el anexo $\mathrm{V}$ (disposición adicional primera); y el establecimiento de un régimen sancionador específico por incumplimientos de la normativa de protección de las aguas frente a la contaminación por nitratos de fuentes agrarias (disposición adicional segunda) ${ }^{3}$.

Por último, la instalación de un "filtro verde" que evite los vertidos de nitratos procedentes de la actividad agrícola parece que se encuentra en un estado de tramitación avanzada; así, el BORM, núm. 63, de 17 de marzo de 2017, publica la Orden por la que se resuelve el expediente de información para la aprobación definitiva del "Proyecto básico para la ejecución de filtro verde en el entorno de la desembocadura de la Rambla del Albujón al Mar Menor", término municipal de Cartagena.

\section{OTRAS MANIFESTACIONES DE LA POLÍTICA Y LEGISLACIÓN AMBIENTAL DE LA ADMINISTRACIÓN REGIONAL EN MURCIA}

\subsection{Educación ambiental}

La sensibilización y concienciación, y, en general, la educación ambiental orientada a la lucha contra el cambio climático son elementos destacados de la Convención Marco de las Naciones Unidas para el Cambio Climático de 1992 y, de nuevo, quedaron reflejados en el Acuerdo de París de diciembre de 2015 (artículo 12.1).

La Fundación Síndrome de Down (Fundown) es una entidad sin ánimo de lucro que tiene entre sus fines "la promoción y realización de todas cuantas actividades contribuyan a la mejora de las condiciones de vida y procuren la plena integración familiar, social y laboral y el desarrollo de una vida normal, de las personas con síndrome de Down, en el marco de solidaridad respecto a otras etiologías que comporten algún tipo de minusvalía". El logro de la plena integración social de personas con discapacidad psíquica mediante la realización de tareas de formación y empleo pasa también por

\footnotetext{
${ }^{3}$ Sobre este tema, véase SORO MATEO, B., "Los errores jurídico-políticos en torno al Mar Menor", Observatorio de Políticas Ambientales 2017 (en prensa).
} 
llevar a cabo actividades propias de la Fundación en un medio natural como el Parque Regional de Carrasco y el Valle.

Ambas partes consideran importante difundir los objetivos y las actividades del Convenio a fin de fomentar el respeto y la sensibilización de la sociedad respecto a la conservación y la mejora del entorno natural y la integración social de los colectivos de personas desfavorecidas.

Por su parte, la Consejería de Agua, Agricultura y Medio Ambiente, a través de la Oficina de Impulso Socioeconómico del Medio Ambiente, tiene entre sus cometidos la puesta en marcha de las estrategias para la mitigación y adaptación al cambio climático en el marco del Plan Estratégico de Adaptación al Cambio Climático. La sensibilización y la concienciación suponen una parte destacable de las estrategias para la lucha contra el cambio climático, puesto que, en el conjunto de emisiones de gases de efecto invernadero, las derivadas del consumo privado de las familias tienen un gran peso.

Este es el origen del Decreto núm. 96/2016, de 14 de septiembre, por el que se regula la concesión directa de una subvención a la Fundación Síndrome de Down (Fundown) para la realización de actividades de educación ambiental en relación a la lucha contra el cambio climático (BORM, núm. 225, de 27 de septiembre). La cuantía es de cuarenta mil euros $(40.000,00 €)$ y estará destinada a la realización de actividades de educación ambiental en la lucha contra el cambio climático desde la consideración de que las personas con discapacidad constituyen un sector sensible y pueden jugar un rol importante como agentes sociales para la divulgación de conocimientos y acciones concretas para la mitigación del cambio climático.

\subsection{Especies exóticas invasoras: el proyecto LIFE "Ripisilvanatura”}

El río Segura se abre paso en muchas zonas de la Región de Murcia por una vegetación única en la Comunidad, el bosque de ribera o de galería (ripisilva), que, sin embargo, se encuentra en grave peligro por la amenaza de especies invasoras que van ocupando progresivamente su espacio. 
EI proyecto LIFE "Ripisilvanatura", que lidera la Confederación Hidrográfica del Segura, pretende controlar la expansión de las especies exóticas invasoras presentes en los márgenes del río Segura y favorecer la colonización del bosque de ribera por especies autóctonas mediante la recuperación y protección del bosque ribereño del curso medio de la cuenca del río, en el tramo que abarca los municipios de Moratalla, Calasparra y Cieza, a través del control de las especies exóticas invasoras (aquellas que se encuentran fuera de su distribución natural y afectan de forma negativa al ecosistema nativo), principalmente en el hábitat catalogado como "prioritario" de los bosques de galería de sauces y chopos.

En especial, en las reservas naturales de Almadenes y Cañaverosa de Murcia la biodiversidad está seriamente amenazada por especies exóticas invasoras como: Opuntia maxima, Washingtonia filifera, Eucalyptus camadulensis, Robinia pseudoacacia, Phoenix canariensis y Arundo donax, entre otras. Estas han colonizado hábitats originales y representan una amenaza para las especies de ribera. Además, el problema de las especies invasoras se ve agravado por la contaminación, el desmonte de tierras para el cultivo directo y una alta incidencia de incendios.

El proyecto plantea crear la infraestructura verde entre las dos reservas naturales ribereñas de la región; aumentar el área de hábitat de bosque de ribera en la zona del proyecto y proteger la flora y fauna pertinentes; eliminar las especies exóticas invasoras; crear una red de custodia de la tierra para la Vega Alta; utilizar la última tecnología para la detección de incendios forestales; y aumentar la conciencia pública ${ }^{4}$.

La actuación, a ejecutar en cinco años, cuenta con un presupuesto total de 2.454.611 euros, de los que la Unión Europea aporta la mitad. Participan como socios colaboradores la Dirección General de Medio Ambiente de la Comunidad de Murcia, la Universidad de Murcia, los ayuntamientos de Calasparra y Cieza y la Asociación de Naturalistas del Sureste (ANSE).

\footnotetext{
${ }^{4}$ En el sitio web del Ministerio de Agricultura y Pesca, Alimentación y Medio Ambiente se puede acceder a información más detallada sobre el proyecto (http://www.mapama.gob.es/es/agua/temas/delimitacion-y-restauracion-del-dominio-publicohidraulico/estrategia-nacional-restauracion-rios/life ripisilvanatura.aspx. Último acceso: 12/04/2017).
} 
El convenio de colaboración entre la Confederación Hidrográfica del Segura y la Administración General de la Comunidad Autónoma de la Región de Murcia, a través de la Consejería de Agua, Agricultura y Medio Ambiente, en calidad de beneficiario asociado, para el desarrollo de las acciones previstas en el proyecto LIFE "Ripisilvanatura" 13/BIO/ES/001407 se publica mediante Resolución de 28 de septiembre de 2016 (BORM, núm. 235, de 8 de octubre de 2017).

\subsection{Hábitats de aves acuáticas en islas de la Región de Murcia}

La planificación de los espacios protegidos Red Natura 2000 en la CARM determinó la definición de catorce áreas de planificación integrada (Orden, de 25 de octubre de 2012, de la Consejería de Presidencia). En concreto, la denominada "API 002" afecta al Área de Planificación Integrada del Mar Menor y Franja Litoral de la Región de Murcia, que incluye las islas de la Región de Murcia, en las que resulta del máximo interés mejorar el estado de conservación de las aves acuáticas. Por ello, mediante Resolución de 28 de septiembre de 2016 (BORM, núm. 235, de 8 de octubre) se publica el Convenio entre la Administración General de la Comunidad Autónoma de la Región de Murcia, a través de la Consejería de Agua, Agricultura y Medio Ambiente, y la Asociación de Naturalistas del Sureste (ANSE) para el desarrollo de un proyecto de marcaje y mejora de hábitats de aves marinas y acuáticas en islas de la Región de Murcia.

Las obligaciones específicas del beneficiario de esta subvención de veinte mil euros $(20.000,00 €)$ quedan establecidas del siguiente modo: a) marcaje e investigación de aves marinas y paseriformes migratorias en islas; b) realización de sendas campañas de anillamiento científico para el estudio del paso migratorio de aves paseriformes durante la primavera y otoño en la isla Grosa; c) determinación de áreas de alimentación e invernada de la colonia de parcela cenicienta en la isla de Las Palomas; d) acciones de restauración de hábitats y eliminación de especies exóticas invasoras; e) arranque y eliminación de ejemplares de especies de flora exótica como acacia y carpobrotus, entre otras; f) evaluación de la acción de depredadores sobre aves marinas; y g) búsqueda de huellas de mamíferos 
con el fin de detectar su posible presencia en las islas con el fin de determinar posibles acciones de control y erradicación.

Por último, se prevén también acciones de divulgación y formación ambiental, en concreto, para las tripulaciones y los responsables de las embarcaciones que realizan visitas al entorno de las islas del litoral de la Región con fines turísticos.

\subsection{Cambio climático}

El cambio climático es uno de los motores de transformación ambiental con mayor impacto sobre la biodiversidad a escala global. Todos los modelos generales de cambio climático predicen para España y, especialmente, para el sureste ibérico un aumento significativo de la temperatura y una disminución de la precipitación, aunque la intensidad y el patrón de esta última está sujeta a diversas interpretaciones y análisis. Estos cambios en los parámetros clave del clima producirán una mayor aridez en el contexto de la Región de Murcia, que afectará a todos los componentes de la biodiversidad, bien sea la flora, la fauna o los ecosistemas en su conjunto.

Se prevén dos cambios principales en la biodiversidad: i) cambios fenológicos, en los ciclos estacionales y en otros patrones temporales; y ii) cambios en la distribución de los organismos, tanto altitudinales como latitudinales. Estas modificaciones deberán materializarse en un contexto de cambio global donde las respuestas de los organismos interaccionan con otras perturbaciones como la degradación de los ecosistemas, la fragmentación o la existencia de barreras a la dispersión. La evaluación certera de las implicaciones en el mantenimiento de los bienes y servicios que la biodiversidad nos ofrece gratuitamente, o en la extinción de especies a escala local y global, precisan de un mayor esfuerzo investigador. En cualquier caso, las redes de espacios protegidos diseñadas hasta el momento como principal estrategia para la conservación de la biodiversidad deben ser revisadas bajo los distintos escenarios climáticos futuros que se plantean desde las instituciones científicas.

Dada la complejidad y magnitud del problema, resulta útil establecer una cierta priorización en las metas que, a corto o medio plazo, deben 
abordarse. La Agencia Europea de Medio Ambiente ha propuesto una serie de indicadores de impacto del cambio climático sobre la biodiversidad que nos permiten establecer dichas prioridades en la obtención de información significativa.

Los principales indicadores de impacto sobre la biodiversidad son: 1) cambios en el área de distribución de especies vegetales clave; 2) cambios equivalentes en la fauna; 3) cambios en los bosques, tanto por decaimiento como por modificaciones en su distribución en altitud y latitud; 4) intensificación de perturbaciones, principalmente incendios y plagas masivas; y 5) cambios en la biodiversidad de áreas críticas (alta montaña, zonas áridas, etc.).

En la Universidad de Murcia se lleva a cabo un proyecto sobre los efectos del cambio climático sobre la biodiversidad en la Región de Murcia: indicadores, impactos y sistemas de alerta. Con él se pretende abordar el máximo número de estos indicadores, especialmente los impactos del cambio climático en: 1) las especies arbóreas silvestres, y singularmente en Pinus halepensis, la más abundante y frecuentemente utilizada en la gestión forestal, para cuyas formaciones deben establecerse niveles de vulnerabilidad que orienten las estrategias de adaptación; 2) las principales especies arbustivas dominantes en nuestros matorrales; 3) las especies clave de zonas áridas (esparto, etc.); y 4) el conjunto de la ornitofauna y algunas otras especies animales potencialmente amenazadas por el cambio climático. En la mayor parte de los casos se establecerán los modelos de distribución actuales y futuros, en función del clima preexistente y los escenarios climáticos más probables, y se delimitarán las áreas más vulnerables ante el cambio climático. Muchas de estas especies son taxones clave en los hábitats considerados en la Red Natura 2000, por lo que se establecerán medidas de adaptación en este sentido, a cuyo efecto se determinarán en el proyecto los principales impactos y los sistemas de alerta.

En este sentido, la Resolución de 11 de octubre de 2016 (BORM, núm. 249, de 26 de octubre) contiene la publicación del convenio de colaboración entre la Administración de la Comunidad Autónoma de la Región de Murcia 
y la Universidad de Murcia para colaborar en el desarrollo del proyecto. La cuantía de la subvención es de cuarenta mil euros $(40.000,00 €)$.

\subsection{Patrimonio natural arbóreo}

La Ley 14/2016, de 7 de noviembre, de Patrimonio Arbóreo Monumental de la Región de Murcia (BORM, núm. 260, de 9 de noviembre de 2017), tiene por objeto "garantizar la protección, conservación, difusión, fomento, investigación y acrecentamiento del patrimonio arbóreo monumental de la Región de Murcia” (art. 1). En este sentido, por patrimonio arbóreo monumental se entiende el "conjunto de árboles cuyas características botánicas de monumentalidad o circunstancias extraordinarias de edad, porte u otros tipos de acontecimientos históricos, culturales, paisajísticos, científicos, de recreo o ambientales ligados a ellos y a su legado, los haga merecedores de protección y conservación" (art. 2.1).

Se exceptúan "Ios ejemplares de especies exóticas invasoras, según las definiciones de la Ley 42/2007, de 13 de diciembre, del Patrimonio Natural y de la Biodiversidad y el Real Decreto 630/2013, de 2 de agosto, por el que se regula el Catálogo español de especies exóticas invasoras" (art. 2.1, apdo. $\left.2 .{ }^{\circ}\right)$.

Este patrimonio arbóreo vivo "comprende también los arbustos u otros ejemplares no arbóreos de dimensiones destacables; los que encierran un importante significado histórico o simbólico y aquellos que recogen tradiciones religiosas o sociales o presentan un alto valor etnoagrario o etnobotánico. Igualmente, deben incluirse en este apartado ejemplares de especies leñosas extremadamente raras, cuya presencia implica un valor científico sobresaliente y aquellos de los que la sociedad disfruta con su contemplación".

Muchos de estos espacios arbolados están en peligro por causas diversas, mayoritariamente achacables a la actividad del ser humano. Estos riesgos "se han visto favorecidos por la falta de conocimiento del número de individuos destacables y de su estado de salud. Se calcula que a lo largo del siglo $\mathrm{XX}$, las anteriores razones han propiciado la pérdida de una parte sustancial del patrimonio arbóreo sobresaliente de la Región de Murcia”. 
Por ello, la Ley establece una serie de medidas de protección que pueden ser arbitradas por la Administración regional (art. 5) y por los ayuntamientos (art. 6). Entre otras de carácter tradicional (planes, subvenciones, autorizaciones, sanciones...), destacamos en estos comentarios la previsión de que la consejería competente en medio ambiente gestionará el Centro de Conservación de la Flora Silvestre, que, además de cumplir los objetivos de recuperación y conservación de la flora silvestre amenazada, asegurará la conservación ex situ y la renovación del germoplasma de los árboles protegidos como monumentales o singulares (art. 16).

\subsection{Pesca en aguas interiores}

La Ley 3/2001, de 26 de marzo, de Pesca Marítima del Estado, y, en el ámbito de la CARM, la Ley $2 / 2007$, de 12 de marzo, de Pesca Marítima y Acuicultura de la Región de Murcia, establecen entre sus fines lograr una explotación equilibrada y responsable de los recursos pesqueros, adaptando el esfuerzo de la flota pesquera a la situación de estos. En concreto, el Plan de Gestión Integral para la conservación de los recursos pesqueros en el Mediterráneo afectados por las pesquerías realizadas con artes de cerco, redes de arrastre y artes fijos y menores, para el período 2013-2017 (Orden AAA/2808/2012, de 21 de diciembre), tiene como finalidad facilitar la consecución de los objetivos biológicos y de gestión sostenible de estas actividades pesqueras en este caladero.

La Orden de 2 de diciembre de 2016, de la Consejería de Agua, Agricultura y Medio Ambiente (BORM, núm. 282, de 5 de diciembre), establece una veda temporal para el ejercicio de la pesca de cerco en aguas interiores de la Región de Murcia durante el período comprendido desde el día 6 de diciembre de 2016 hasta el día 6 de enero de 2017, ambos inclusive (art. 1).

Por lo que se refiere a la pesca de arrastre, por otro lado, la Orden de 28 de febrero de 2017 establece un período de veda temporal para el ejercicio de la pesca de arrastre en aguas interiores de la Región de Murcia (BORM, núm. 57, de 10 de marzo de 2017) desde el día 13 de mayo hasta el día 11 de junio de 2017, ambos inclusive. 


\subsection{Residuos: la aprobación del nuevo Plan de Residuos de la Región de Murcia 2016-2020}

La Resolución de 29 de diciembre de 2016 (BORM, núm. 301, de 30 de diciembre) hace pública la aprobación del Plan de Residuos de la Región de Murcia.

El Plan de Residuos de la Región de Murcia 2016-2020 viene a sustituir el Plan de Residuos Urbanos y de Residuos no Peligrosos 2001-2006 y supondrá una inversión de más de 42 millones de euros durante su período de ejecución. Con este plan se cumple, en el ámbito territorial de la Región de Murcia, lo establecido en la Ley 22/2011, de 28 de julio, de residuos y suelos contaminados, cuyo objeto es regular la gestión de los residuos mediante el impulso de medidas que prevengan su generación y mitiguen los impactos adversos asociados a su generación y gestión, mejorando la eficiencia en el uso de los recursos.

Su período de vigencia se justifica en que 2020 es el año en que finaliza el plazo de la Directiva de Residuos y de la Ley estatal de Residuos para el cumplimiento de los objetivos establecidos en ellas.

El Plan incorpora un análisis sobre la situación de la prevención y gestión de residuos, así como una exposición de las medidas para facilitar la reutilización, el reciclado, la valorización y la eliminación de los residuos. Entre sus objetivos se encuentra reducir el 10\% (unas 66.630 toneladas) de los residuos domésticos, para alcanzar las 600.057 toneladas en 2020. Otro reto consiste en incrementar la recogida separada de residuos de envases domésticos, de forma que en 2020 se consiga como mínimo que el $70 \%$ del reciclado proceda de residuos de envases recogidos de forma separada en la Región de Murcia. También se plantea alcanzar el 50\% de preparación para la reutilización y el reciclado en 2020 , de los cuales un $2 \%$ corresponderá a la preparación para la reutilización, fundamentalmente de residuos textiles, muebles y otros residuos susceptibles de ser preparados para su reutilización. 
En definitiva, la intención del Gobierno regional es tratar de convertir los residuos en recursos, subrayando las nuevas oportunidades de inversión a partir de nuevas líneas de investigación y del desarrollo de nuevas tecnologías. El nuevo Plan aborda asimismo la lucha contra el fraude y la competencia desleal que demandan los gestores autorizados. Además, ya en el primer año de vigencia se prevén actuaciones para la adecuación y mejora de los sistemas básicos de gestión de residuos a fin de mejorar los sistemas e instalaciones para tratamiento térmico, aprovechamiento energético y depósito en vertedero de residuos domésticos y asimilados, incluyendo trabajos de seguimiento del llenado de vertederos, así como las operaciones de sellado y restauración.

El contenido completo del Plan puede consultarse en http://www.carm.es/web/pagina?IDCONTENIDO=128897\&IDTIPO=60

\subsection{Directiva Hábitats: modelos normalizados de comunicación previa de usos y actividades}

El orden de prioridad de los cincuenta lugares de importancia comunitaria (LIC) de la Región de Murcia para su declaración como zonas de especial conservación (ZEC) se aprobó mediante Acuerdo del Consejo de Gobierno de 6 de julio de 2012, de conformidad con el artículo 4.4 de la Directiva Hábitats. Por su parte, la Orden de 25 de octubre de 2012, del consejero de Presidencia, definió, para el conjunto de los espacios protegidos de la Región de Murcia, catorce áreas de planificación integrada (API) con el fin de que los diferentes regímenes aplicables en función de cada categoría conformaran un todo coherente, en aplicación del artículo 29.2 de la Ley 42/2007, de 13 de enero.

El artículo 10 del Decreto 55/2015, de 17 de abril, de Declaración de Zonas Especiales de Conservación y Aprobación del Plan de Gestión Integral de los espacios protegidos Red Natura 2000 del Noroeste de la Región de Murcia, regula el procedimiento de comunicación previa por el que los interesados pondrán en conocimiento del órgano gestor de la Red Natura 2000 sus datos identificativos acompañando la documentación e 
información exigible para la ejecución de determinadas actividades expresamente contempladas en las regulaciones del Plan de Gestión Integral. Por otro lado, se establece la obligación de aprobar por orden del consejero competente en materia de medio ambiente los modelos normalizados de comunicación previa.

Respecto de las restantes áreas de planificación integrada, se prevé la aprobación sucesiva de los correspondientes planes, que en la actualidad se encuentran en distintas fases de tramitación. Todos contemplan determinados supuestos respecto de los cuales los interesados deberán someter ciertas actividades a comunicación al órgano gestor de los espacios protegidos.

Por ello, mediante Orden de 16 de enero de 2017, de la Consejería de Agua, Agricultura y Medio Ambiente (BORM, núm. 16, de 21 de enero de 2017), se aprueban los modelos normalizados de comunicación previa de usos y actividades de los planes de gestión y su inmediata puesta a disposición de los ciudadanos afectados por las regulaciones que se contengan en los planes de todo el ámbito regional en el momento de la vigencia de cada uno de ellos, y sin perjuicio de la ulterior ampliación de los modelos normalizados de comunicación para el desarrollo de nuevas actividades que, de forma singular, se vayan añadiendo en las regulaciones de cada uno de los planes que se aprueben.

\subsection{Participación ambiental: de nuevo sobre la destrucción del Mar Menor}

El extraordinario valor ambiental de la laguna del Mar Menor y el proceso de deterioro que sufre la calidad de sus aguas, que hemos sintetizado supra en esta misma crónica, condujeron a la creación de un comité de asesoramiento que integrara a los grupos científicos de las universidades y organismos de investigación que desarrollan líneas de trabajo relacionadas con el Mar Menor. La constitución del Comité de Asesoramiento Científico del Mar Menor se llevó a cabo por la Orden de 29 de julio de 2016, de la Consejería de Agua, Agricultura y Medio Ambiente, que se dotaba de esta 
manera de un asesoramiento científico adecuado para la selección de las acciones necesarias para encarar el problema.

No se debe olvidar, sin embargo, que en este espacio convergen múltiples usos y aprovechamientos, principalmente turísticos, recreativos y pesqueros, así como un importante aprovechamiento agrícola en su entorno. La laguna constituye, por tanto, un factor clave en los planes de desarrollo regional, con relevante significación en términos de desarrollo económico, turístico y de empleo. La problemática ambiental que hoy soporta el Mar Menor tiene importantes repercusiones económicas y sociales que no se pueden soslayar, sino que deben integrarse en la consideración global del problema y en las soluciones que se adopten.

Por todo ello, el Gobierno regional ha considerado preciso la creación de un foro de participación social en el que tengan voz ese conjunto de intereses económicos, sociales y vecinales. Se pretende favorecer el diálogo y la búsqueda de soluciones de consenso para solventar los problemas ambientales del Mar Menor y su área de influencia. Los representantes sociales podrán expresar a través de este comité las implicaciones que la crisis ambiental de la laguna tiene para los distintos sectores de actividad y colectivos afectados, y aportarán la perspectiva necesaria para un enfoque integral de las actuaciones que deben acometerse.

Su regulación sigue los límites establecidos por el artículo 24.3, apartado

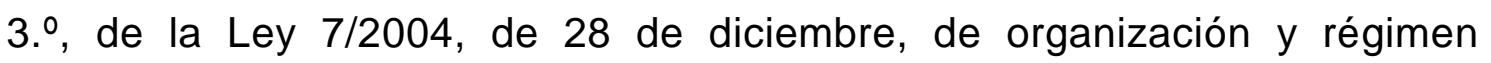
jurídico de la Administración Pública de la Comunidad Autónoma de la Región de Murcia, que dispone que aquellos órganos colegiados que no tengan competencias decisorias, de propuesta o emisión de informes preceptivos que deban servir de base a decisiones de otros órganos administrativos, ni competencias de seguimiento 0 control de las actuaciones de otros órganos de la Administración Pública de la Comunidad Autónoma, tendrán el carácter de grupos o comisiones de trabajo y podrán ser creados por las consejerías interesadas. Sus acuerdos no podrán tener trascendencia jurídica directa frente a terceros. Además, de acuerdo con el artículo 23.5 de la misma Ley, en la composición de los órganos colegiados pueden participar organizaciones representativas de intereses sociales, así 
como otros miembros que se designen por las especiales condiciones de experiencia o conocimientos que concurran en ellos, en atención a la naturaleza de las funciones asignadas a tales órganos.

Con esta base legal, se crea mediante la Orden de 28 de febrero de 2017, de la Consejería de Agua, Agricultura y Medio Ambiente, el Comité de Participación Social del Mar Menor (BORM, núm. 52, de 4 de marzo de 2017), con una composición plural (art. 3) y unas normas de funcionamiento flexibles (art. 4), como es propio de un órgano de estas características.

\subsection{Flora: Plan de Recuperación del Garbancillo de Tallante}

Las escasas poblaciones naturales existentes, la superficie extremadamente reducida que ocupa y las diversas presiones antrópicas a las que se ve sometido el garbancillo de Tallante determinan que su recuperación sea prioritaria en la gestión y conservación de las especies amenazadas de la flora silvestre de la Región de Murcia.

Esta especie está clasificada en la categoría de "en peligro de extinción" en el Catálogo Español de Especies Amenazadas (Real Decreto 139/2011, de 4 de febrero, para el desarrollo del Listado de Especies Silvestres en Régimen de Protección Especial y del Catálogo Español de Especies Amenazadas) y en el Catálogo Regional de Flora Silvestre Protegida de la Región de Murcia, y con el ámbito territorial establecido en este (anexo I del Decreto núm. 50/2003, de 30 de mayo, por el que se crea el Catálogo Regional de Flora Silvestre Protegida de la Región de Murcia y se dictan normas para el aprovechamiento de diversas especies forestales).

En consecuencia, el Decreto núm. 12/2017, de 22 de febrero, aprueba el Plan de Recuperación del Garbancillo de Tallante (Astragalus nitidiflorus) (BORM, núm. 51, de 3 de marzo de 2017). 